PROCEEDINGS OF THE

AMERICAN MATHEMATICAL SOCIETY

Volume 49, Number 2, June 1975

\title{
A NOTE ON JONES' FUNCTION $K$
}

\author{
JOHN ROSASCO
}

ABSTRACT. For each point $x$ of a continuum $M, F$. B. Jones [5, Theorem 2] defines $K(x)$ to be the closed set consisting of all points $y$ of $M$ such that $M$ is not aposyndetic at $x$ with respect to $y$. Suppose $M$ is a plane continuum and for any positive real number $\epsilon$ there are at most a finite number of complementary domains of $M$ of diameter greater than $\epsilon$. In this paper it is proved that for each point $x$ of $M$, the set $K(x)$ is connected.

A continuum $M$ ( nondegenerate metric space that is compact and connected) is said to be aposyndetic at a point $p$ of $M$ with respect to a point $q$ of $M$ if there exist an open set $W$ and a continuum $H$ in $M$ such that $p \in W \subset H \subset M-\{q\}$.

Throughout this paper $S$ is the set of points of a simple closed surface (2-sphere).

Definition. Let $M$ be a continuum in $S$ and let $x$ and $y$ be distinct points of $M$. The set $S-M$ is said to be folded around $x$ with respect to $y$ if there exist two monotone descending sequences of circular regions $U_{1}, U_{2}, U_{3}, \ldots$ and $V_{1}, V_{2}, V_{3}, \ldots$ in $S$ centered on and converging to $x$ and $y$ respectively such that $\mathrm{Cl} U_{1} \cap \mathrm{Cl} V_{1}=\varnothing\left(\mathrm{Cl} U_{1}\right.$ is the closure of $U_{1}$ ), and there exists a sequence of mutually exclusive sets $X_{1}, X_{2}, X_{3}$, ... in $S-M$ having the following properties. For each positive integer $i$, the set $X_{i}$ is the union of two intersecting arc-segments (open arcs) $I_{i}$ and $T_{i}$ such that

(1) $I_{i} \cap T_{i}$ is connected,

(2) $I_{i}$ is contained in $\operatorname{Bd~} U_{i}\left(\operatorname{Bd} U_{i}\right.$ is the boundary of $\left.U_{i}\right)$ and has endpoints $a_{i}$ and $b_{i}$ in $M$,

(3) the sets $\mathrm{Cl} U_{i+1}$ and $\left(a_{i}\right.$-component of $M-V_{i}$ ) are disjoint,

Presented to the Society, November 23, 1974; received by the editors February 17, 1974.

AMS (MOS) subject classifications (1970). Primary 54F 15, 54F 20, 54A05; Secondary 54F 25.

Key words and phrases. Jones' function $K$, aposyndesis, folded complementary domain, nonlocally connected plane continua. 
(4) $T_{i}$ is contained in $S-\mathrm{Cl}\left(V_{i} \cup U_{i+1}\right)$ and has two distinct endpoints in $\operatorname{Bd} V_{i}$,

(5) $T_{i} \cup \mathrm{Bd} V_{i}$ contains a simple closed curve $S_{i}$ that separates $a_{i}$ from $b_{i}$ in $S$.

Theorem. If $M$ is a continuum in $S$ and for any positive real number $\epsilon$ there are at most a finite number of complementary domains of diameter greater than $\epsilon$, then for each point $x$ of $M$, the set $K(x)$ is connected.

Proof. Assume $K(x)$ is not connected. Let $y$ be a point of $K(x)$ that does not belong to the $x$-component of $K(x)$. There exists an open disk $R$ such that $y$ belongs to $R$, the disk $\mathrm{Cl} R$ is contained in $S-\{x\}$, and $M$ is aposyndetic at $x$ with respect to each point of $M \cap \operatorname{Bd} R[6$, Theorem 49, p. 17 and Theorem 13, p. 170].

Since $M$ is not aposyndetic at $x$ with respect to $y, S-M$ is folded around $x$ with respect to $y\left[4\right.$, Theorem 2]. Let $U_{1}, U_{2}, U_{3}, \ldots, V_{1}, V_{2}$, $V_{3}, \cdots$, and $X_{1}, X_{2}, X_{3}, \ldots$ be the sequences, as described in the definition, which indicate that $S-M$ is folded around $x$ with respect to $y$. Assume without loss of generality that $\mathrm{Cl} U_{1} \cap \mathrm{Cl} R=\varnothing$ and $\mathrm{Cl} V_{1} \subset R$.

For each positive integer $n$, let $A_{n}$ and $B_{n}$ denote the $a_{n}$-component and the $b_{n}$-component of $M-R$ respectively. According to $[1$, Lemma and the third paragraph in the proof of Theorem 1], we can assume without loss of generality that there exist disjoint arc-segments $C$ and $E$ in $\mathrm{Bd} R$ such that for each $n, A_{n}$ meets both $C$ and $E$ and $B_{n}$ meets both $C$ and $E$. For each $n$, let $c_{n}$ and $e_{n}$ be points of $A_{n} \cap C$ and $A_{n} \cap E$ respectively. Assume without loss of generality that for each $n, A_{n+1}$ separates $A_{n}$ from $A_{n+2}$ in $S-R$ [6, Theorem 28, p. 156]. For each $n$, since the arc-segment $I_{n}$ is contained in $S-M, B_{n+1}$ also separates $A_{n}$ from $A_{n+2}$ in $S-R$.

The sequence $c_{1}, c_{2}, c_{3}, \ldots$ converges to a point $v_{1}$ of $M \cap \mathrm{Cl} C$ and $e_{1}, e_{2}, e_{3}, \ldots$ converges to a point $v_{2}$ of $M \cap C l E$. The points $v_{1}$ and $v_{2}$ are distinct; for otherwise, it would follow that $M$ is not aposyndetic at $x$ with respect to $v_{1}[1$, the fourth paragraph in the proof of Theorem 1].

Since $M$ is aposyndetic at $x$ with respect to each point of $\mathrm{Bd} R$, there exist subcontinua $H_{1}$ and $H_{2}$ of $M$ and circular regions $G_{1}$ and $G_{2}$ such that $\mathrm{Cl} G_{1} \cap \mathrm{Cl} G_{2}=\varnothing$ and such that for $n=1$ and $n=2$, the region $G_{n}$ contains $v_{n}, H_{n} \cap \mathrm{Cl} G_{n}=\varnothing$, and the point $x$ is in the interior of $H_{n}$ relative to $M$. There is a circular region $W$ that contains $x$ such that $\mathrm{Cl} W \cap \mathrm{Cl}\left(G_{1} \cup G_{2}\right)=\varnothing$ and $W \cap M$ is contained in $H_{1} \cap H_{2}$.

Assume without loss of generality that $\mathrm{ClC}$ is in $G_{1}, \mathrm{Cl} E$ is in $G_{2}$, 
and $\mathrm{Cl}_{1}$ is in $W$. Let $\epsilon=\operatorname{dist}[W, R]$. Since there are at most a finite number of complementary domains of diameter greater than $\epsilon$, there exist integers $m$ and $n$ such that $T_{m}$ and $T_{n}$ belong to the same complementary domain of $M$.

Let $T_{m}^{\prime}$ be the component of $T_{m}-R$ that contains $T_{m} \cap I_{m}$ and let $T_{n}^{\prime}$ be the component of $T_{n}-R$ that contains $T_{n} \cap I_{n}$. Since $A_{m} \cup B_{m} \cup C$ $\cup E$ separates $I_{m}$ from $R$ in $S$, we know that $T_{m}^{\prime}$ intersects $\left(G_{1} \cup G_{2}\right)$. Note also that $T_{m}^{\prime}$ intersects both $G_{1}$ and $G_{2}$, since otherwise the union of $T_{m}^{\prime}$ and a component of $\operatorname{Bd}\left(G_{1} \cup G_{2}\right)$ would separate $a_{m}$ from $b_{m}$ in $S$ [6, Theorem 32, p. 181], and this would contradict the existence of $H_{1}$ and $H_{2}$. Similarly $T_{n}^{\prime}$ intersects both $G_{1}$ and $G_{2}$.

Since $T_{m}^{\prime}$ and $T_{n}^{\prime}$ belong to the same complementary domain of $M$, there is an arc $A$ in $S-M$ that intersects both $T_{m}^{\prime}$ and $T_{n}^{\prime}$. Let $K=T_{m}^{\prime} \cup T_{n}^{\prime} \cup$ $A \cup \mathrm{Bd} G_{1}$ and let $H=T_{m}^{\prime} \cup T_{n}^{\prime} \cup A \cup \mathrm{Bd} G_{2}$. The set $K \cup H$ separates $a_{m}$ from $b_{m}$ in $S[6$, Theorem 32, p. 181]. Since $K \cap H$ is connected, we can assume without loss of generality that $K$ separates $a_{m}$ from $b_{m}$ in $S$ [6, Theorem 20, p. 173]. Since $H_{1}$ contains $\left\{a_{m}, b_{m}\right\}$ and misses $K$, this contradicts the fact that $H_{1}$ is a continuum. It follows that $K(x)$ must be connected.

As a consequence of this theorem, we have the following result announced by C. L. Hagopian in [3].

Corollary. $K(x)$ is connected for eacb point $x$ of a plane continuum that has only finitely many complementary domains.

Continua that satisfy the hypothesis of our theorem are called E-continua by G. T. Whyburn. In [7, Theorem 4.4, p. 113] several conditions are given that characterize local connectivity in these spaces. It is proved in [2] that semi-aposyndetic $E$-continua are arcwise connected.

Example. The set $K(x)$ may fail to be connected for a point $x$ of a plane continuum that is not an $E$-continuum. To see this, let $C$ be the Cantor discontinuum and define $M$ to be the quotient space

$$
C \times[0,1] / C \times\{0,1\} .
$$

Let $y$ be the separating point of $M$. Then for each point $x$ of $M-\{y\}$, the set $K(x)=\{x, y\}$.

\section{REFERENCES}

1. C. L. Hagopian, A cut point theorem for plane continua, Duke Math. J. 38 (1971), 509-512. MR 44 \#2204. 
2. C. L. Hagopian, Arcwise connectivity of semi-aposyndetic plane continua, Pacific J. Math. 37 (1971), 683-686.

3. - Concerning Jones's function K, Notices Amer. Math. Soc. 19 (1972), A-779. Abstract \#698-G2.

4. F. B. Jones, A characterization of a semi-locally-connected plane continuum, Bull. Amer. Math. Soc. 53 (1947), 170-175. MR 8, 397.

5. - Concerning non-aposyndetic continua, Amer. J. Math. 70 (1948), 403-413. MR 9, 606.

6. R. L. Moore, Foundations of point set theory, rev. ed., Amer. Math. Soc. Colloq. Publ., vol. 13, Amer. Math. Soc., Providence, R. I., 1962. MR 27 \# 709. 7. G. T. Whyburn, Analytic topology, Amer. Math. Soc. Colloq. Publ., vol. 28, Amer. Math. Soc., Providence, R. I., 1942. MR 4, 86.

DEP ARTMENT OF MATHEMATICS, CALIFORNIA STATE UNIVERSITY, SACRAMENTO, CALIFORNIA 95819

Current address: 6460 18th Avenue, Sacramento, California 95820 